\title{
AN IN PLANTA INDUCED GENE OF PHYTOPHTHORA INFESTANS CODES FOR UBIQUITIN
}

\author{
C.M.J. PIETERSE, E.P. RISSEEUW and L.C. DAVIDSE \\ Department of Phytopathology \\ Agricultural University of Wageningen \\ P.O. Box 8025 \\ 6700 EE Wageningen \\ The Netherlands
}

\begin{abstract}
An in planta induced gene of Phytophthora infestans (the causal organism of potato late blight) was isolated from a genomic library by differential hybridization using labelled cDNA derived from poly $(\mathrm{A})^{*}$ RNA of $P$. infestans grown in vitro and labelled cDNA made from potato- $P$. infestans interaction poly $(\mathrm{A})^{+}$RNA as probes. Sequence analysis showed that the gene codes for ubiquitin, a highly conserved protein which plays an important role in several cellular processes. The structure of the polyubiquitin gene is consistent with the stucture of other known polyubiquitin genes. Northern and Southern blot analyses revealed that the polyubiquitin gene is a member of a multigene family of which all genes show induced expression in planta.
\end{abstract}

\section{Introduction}

Potato late blight caused by the fungus Phytophthora infestans (Mont.) de Bary (Oomycetes) is one of the most important diseases of potato. Leaves and tubers of susceptible cultivars become readily infected by this pathogen. The fungus spreads rapidly through the plant tissue causing a destructive necrosis.

It can be assumed that the establishment of a pathogenic relation between the potato plant and $P$. infestans involves mutual interference in cellular processes of each partner. Defence responses of host tissue being colonized are relatively well studied [1-3], but nothing is known about the molecular basis underlying pathogenicity of the fungus. In order to gain more insight in the molecular processes involved we are studying the gene expression of $P$. infestans during pathogenesis on potato by differential screening of a genomic library of $P$. infestans DNA. Several differentially hybridizing clones containing putative in planta induced genes of $P$. infestans were isolated. The coding region of one of these genes was mapped on the lambda clone and completely sequenced. The sequence appeared to code for ubiquitin, a highly conserved protein in eukaryotic organisms which is reported to be involved in several important cellular processes such as intracellular protein breakdown, maintainance of chromatin structure, regulation of gene expression and modification of cell surface receptors (for reviews see [4$6]$ ). The significance of the in planta induced expression of this gene will be discussed.

\section{Differential screening of the genomic library of $P$. infestans DNA}

Differential screening of the genomic library with fungal and interaction cDNA is performed under non-saturating conditions. Under these conditions the intensity of each obtained signal corresponds with the abundancy of a particular cDNA in the probe. A stronger signal obtained 
after hybridization with interaction cDNA compared to the signal obtained after hybridization with fungal cDNA indicates a higher abundancy of those cDNA's which are complementary to the DNA in the hybridyzing genomic lambda clone. Since the filters are hybridized with equal amounts of labeled cDNA the procedure even underestimates the difference in abundancy because the quantity of fungal derived cDNA present in the interaction cDNA probe is much less than in the fungal cDNA probe.

The genomic library of $P$. infestans DNA in phage lambda EMBL3 was differentially screened using equal amounts $\left(10^{7} \mathrm{cpm}\right)$ of ${ }^{32} \mathrm{P}$-labeled $\mathrm{CDNA}$ made from poly $(\mathrm{A})^{+} \mathrm{RNA}$ of the fungus grown in vitro and ${ }^{32} \mathrm{P}$-labeled $\mathrm{cDNA}$ derived from interaction poly $(\mathrm{A})^{+} \mathrm{RNA}$ as probes. Several clones giving a relatively strong signal after hybridization with labeled interaction cDNA and a relatively weak or no signal after hybridization with labeled cDNA of the fungus grown in vitro were isolated. One of these clones was purified after a second round of hybridization. Southem analysis of various restriction fragments of the isolated lambda clone with interaction cDNA as probe revealed a strongly hybridizing SstI-fragment of $3.2 \mathrm{~kb}$ in length. This fragment containing a putative in planta induced gene was subcloned in pTZ19U and a map of restriction sites was constructed from comparisons of single and multiple restriction enzyme digests of the obtained plasmid pUB-S. The approximate location of the coding region of the differentially expressed gene (closed bar in Fig. 1a) was determined by Southem blot analysis using interaction cDNA as probe.
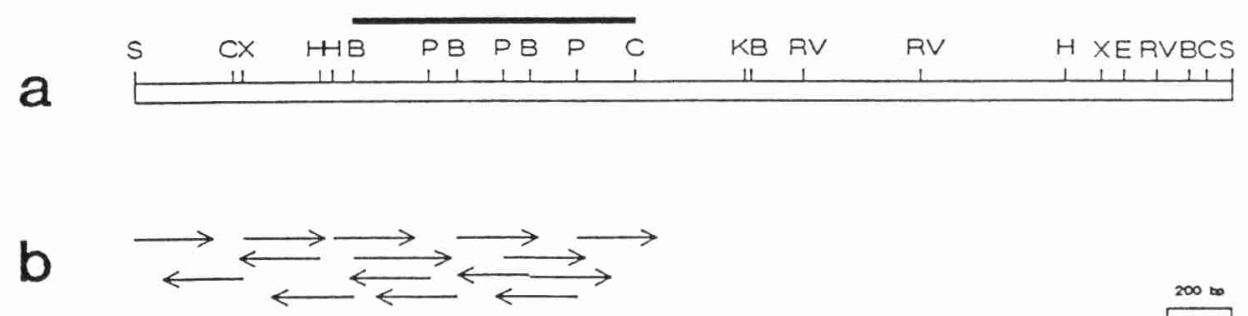

Figure 1. a) Restriction map of the $3.2 \mathrm{~kb}$ Sst I insert of pUB-S. The closed bar indicates the approximate location of the coding region of the differentially expressed gene as determined by Southern hybridization using labeled interaction cDNA as probe. b) The sequencing strategy is indicated by arrows. $\mathrm{B}=B g l \mathrm{II} ; \mathrm{C}=C l a \mathrm{I} ; \mathrm{E}=E \operatorname{coRI} ; \mathrm{H}=H$ ind III; $\mathrm{K}=K p n \mathrm{I} ; \mathrm{P}=P v u \mathrm{II} ; \mathrm{S}=S s t \mathrm{I}$; $\mathrm{X}=$ XhoI; RV=EcoRV.

\section{Characterization of the in planta induced gene of $P$. infestans}

Several fragments of the $3.2 \mathrm{~kb}$ SstI-insert of pUB-S were subcloned and the sequence of 1632 nt was determined by dideoxy sequencing on both strands of various overlapping clones (Pieterse et al, submitted). The sequencing strategy is summarized in Fig. 1b. An open-reading frame of $687 \mathrm{nt}$ containing three almost identical $228 \mathrm{nt}$ repeats was found in the $1632 \mathrm{nt}$ sequence. Comparison of the $687 \mathrm{nt}$ sequence with the sequence databank revealed that the sequence codes for polyubiquitin. The coding region encodes three ubiquitin units with a length of 76 amino acids in a head-to-tail arrangement followed by an extra asparagine residue at the carboxy-terminal end (Fig. 2). Although the ubiquitin-coding repeats within the gene differ up to 8 out of 228 bases, they code for identical amino acid sequences. Only 5 amino acids of the 76 amino acid ubiquitin sequence differ from the sequence of human, chicken and yeast ubiquitin. 


\section{QLEDGRTLSD YNIQKESTLH LVLRLRGGN *}

Figure 2. Predicted amino acid sequence of the cloned ubiquitin gene of $P$. infestans. Arrows indicate the start of the first, second and third ubiquitin encoding repeat.

\section{In planta induced expression of $P$. infestans ubiquitin genes}

Northern blot analysis of RNA isolated from the in vitro grown mycelium, using the two 228 bp $P v u I I-$ fragments from the ubiquitin coding region of pUB-S as probe, shows five bands of approximately $640,870,1100,1650$ and 2150 nucleotides in length. The size differences between the transcripts are such that each transcript appears to contain a different number of ubiquitin repeats $(2,3,4,6$ and 8 respectively). This indicates that the genome of $P$. infestans contains multiple copies of ubiquitin encoding genes of different lengths. Southern blot analysis of digested genomic DNA in which the ubiquitin encoding 228 bp $P v u$ II-fragments of pUB-S were used as a probe, confirmed that the identified ubiquitin gene belongs to a multigene family.

The differential expression of the ubiquitin genes was confirmed by Northem blot analysis of equal amounts of interaction and fungal poly $(\mathrm{A})^{+}$RNA. RNA isolated from colonized leaf tissue and RNA isolated from the fungus grown in vitro give rise to comparable signals upon hybridization with the ubiquitin coding region. Although the amount of fungal RNA as a percentage of the total RNA obtained from the interaction is difficult to measure, it most certainly is very low $(5-10 \%)$. Taking the strong under representation of fungal RNA in the interaction RNA mixture in consideration, a several fold higher expression of all the ubiquitin encoding genes during growth of the fungus in planta could be deduced from the Northern blot.

\section{Discussion}

Ubiquitin is one of the most highly conserved proteins known to date (for reviews see [46]). The 76 amino acid protein occurs in all eukaryotic cells, either free or covalently attached to proteins in the cytosol, plasma membrane or to chromosomal histones. Ubiquitin has been shown to play a key role in several important cellular processes such as the selective degradation of intra-cellular proteins, maintainance of chromatin structure, regulation of gene expression and modification of cell surface receptors. Genes encoding ubiquitin have been characterized from a variety of organisms such as yeast [7], human [8], chicken [9]. In each case, ubiquitin is encoded by one or more polyubiquitin genes which consist of direct repeats of the 76 amino acid coding units. The last repeat at the 3 ' end of the polyubiquitin gene is usually followed by an extra amino acid residue which is not conserved among different species. Although the unique structure of polyubiquitin genes has been conserved in evolution, considerable variation exists in the number of repeats within each polyubiquitin gene and the 
number of polyubiquitin encoding loci in the genome.

Induced expression of polyubiquitin genes by heatshock or other types of stress has been observed in a number of organisms $[9,10]$. This facilitates an increased production of ubiquitin monomers for the ubiquitin-mediated degradation of abnormal proteins which arise during stress. The induced expression of ubiquitin genes in $P$. infestans during colonization of potato leaves may reflect the highly-active metabolic state of the mycelium in the host tissue. During exponential growth in vitro however, the mycelium is also in a highly-active metabolic state but the ubiquitin encoding genes show no induced expression. This implies that the host environment specifically induces the expression of the ubiquitin genes. In view of the regulatory function of ubiquitin in gene expression it is tempting to speculate that induction of the $P$. infestans polyubiquitin genes may lead to expression of genes involved in pathogenicity.

\section{References}

1. Coolbear, T. and Threlfall, D.R. (1985) 'A comparison of the sites of phytoalexin accumulation and of biosynthetic activity in potato tuber tissue inoculated with biotic elicitors', Phytochemistry 24 , 2219-2224.

2. Kombrink, E., Schröder, M. and Hahlbrock, K. (1988) 'Several "pathogenesis-related" proteins in potato are 1,3-B-glucanase and chitinase', Proc. Natl. Acad. Sci. USA 85, 782-786.

3. Cuypers, B., Schmelzer, E. and Hahlbrock, K. (1988) 'In situ localization of rapidly accumulated phenylalanine ammonia-lyase mRNA around penetration sites of Phytophthora infestans in potato leaves', Molecular Plant-Microbe Interactions 1, 157-160.

4. Monia, B.P., Ecker, D.J. and Crooke, S.T. (1990) 'New perspectives on the structure and function of ubiquitin', Bio/technology 8, 209-215.

5. Finley, D. and Varshavsky, A. (1985) 'The ubiquitin system: Functions and mechanisms', Trends Biochem. Sci. 10, 343-347.

6. Hershko, A. (1988) 'Ubiquitin-mediated protein degradation', J. Biol. Chem. 263, 15237-15240.

7. Ozkaynak, E., Finley, D., Solomon, M-J. and Varshavsky, A. (1987) 'The yeast ubiquitin genes: A family of natural gene fusions', EMBO J. 6, 1429-1439.

8. Wiborg, O., Pedersen, M.S., Wind, A., Berglund, L.E., Marcker, K.A. and Vuust, J. (1985) 'The human ubiquitin gene family: Some genes contain multiple directly repeated ubiquitin coding sequences', EMBO J. 4: 755-759.

9. Bond, U. and Schlesinger, M.J. (1985) 'Ubiquitin is a heat shock protein in chicken embryo fibroblasts', Mol. Cell Biol. 5, 949-959.

10. Muller-Taubenberger, A., Hagmann, J., Noegel, A. and Gerisch, G. (1988) 'Ubiquitin gene expression in Dictyostelium is induced by heat and cold shock, cadmium, and inhibitors of protein synthesis', J. Cell Sci. 90, 51-58. 